\title{
Mapeo y caracterización servicio ecosistémico de provisión y recreación en la cordillera de Nahuelbuta $\left(3^{\circ} 30^{\prime \prime}\right.$ - $38^{\circ} 00^{\prime \prime}$ Sur)
}

\section{The Mapping and Characterization of Product and Recreation Ecosystem Services in the Nahuelbuta Mountain Range}

\author{
Kimberly Arriagada, Mauricio Aguayo Arias
}

\begin{abstract}
Resumen
Los servicios ecosistémicos son los beneficios que las personas obtienen de los ecosistemas naturales. La caracterización y cuantificación de los servicios ecosistémicos contribuyen a la gestión sustentable de los recursos naturales. Los bosques son ecosistemas que otorgan múltiples servicios, entre los que se destacan servicios de provisión de productos forestales no maderables y servicios culturales de recreación. En este contexto el estudio se enfocó en el análisis y evaluación de los servicios ecosistémicos de la Cordillera de Nahuelbuta a través del mapeo y cuantificación de dichos servicios. Se analizó cómo influye el grado de naturalidad de las formaciones boscosas en la generación de estos servicios. Se estimó el stock de productos forestales no maderables concentrándose en frutos, semillas y hongos. Los resultados arrojan que el área alberga hasta 36 especies que proveen productos forestales no maderables en las formaciones boscosas de Bosque mixto templado de Nahuelbuta de Nothofagus dombeyi y N. obliqua y el Bosque laurifolio templado costero de Aextoxicon punctatum y Laurelia sempervirens. En relación al servicio de recreación se estimó un alto potencial de recreación y ecoturismo en la zona, en especial los sectores de Caramávida, Trongol y Parque Nacional Nahuelbuta los cuales presentan una adecuada combinación de atributos para la recreación tales como recursos singulares, belleza escénica, accesibilidad y capacidad de atracción turística. Los resultados revelan la necesidad de una urgente y adecuada protección de aquellas áreas núcleos en donde se concentra la mayor provisión de los servicios ecosistémicos analizados.
\end{abstract}

Palabras clave: servicios ecosistémicos, recreación, Productos Forestales No Maderables, Cordillera de Nahuelbuta, Chile.

\begin{abstract}
Ecosystem services are the benefits that people derive from natural ecosystems. The characterization and quantification of ecosystem services contribute to the sustainable management of natural resources. Forests are ecosystems that provide multiple services, including non-timber forest product provision services and cultural recreation services. In this context, the study focused on the analysis and evaluation of the ecosystem services of the Cordillera de Nahuelbuta through the mapping and quantification of these services. We analyzed how the degree of naturalness of forest formations influences the generation of these services. The stock of non-timber forest products was estimated by concentrating on fruits, seeds and fungi. The results show that the area harbors up to 36 species that provide non-timber forest products in the forested areas of the temperate mixed forest of Nahuelbuta of Nothofagus dombeyi and N. obliqua and the coastal temperate laurifolius of Aextoxicon punctatum and Laurelia sempervirens. In relation to the recreation service, there was a high potential for recreation and ecotourism in the area, especially the sectors of Caramávida, Trongol and Nahuelbuta National Park which present an appropiate combination of attributes for recreation such as singular resources, scenic beauty, accessibility and tourism attractiveness. The results reveal the need for an urgent and adequate protection of those core areas where the largest provision of the analyzed ecosystem services is concentrated.
\end{abstract}

Keywords: Ecosystem services, recreation, non-timber Forest products, cordillera de Nahuelbuta, Chile.

Recibido el 04 de julio de 2017, aceptado el 17 de octubre de 2017

Facultad de Ciencias Ambientales, Centro EULA-Chile. Universidad de Concepción, Concepción, Chile. Email: maaguayo@udec.cl 


\section{Introducción}

En las últimas décadas, se han introducido cambios sin precedentes en los ecosistemas con el fin de satisfacer las crecientes demandas de alimento, agua, fibra $\mathrm{y}$ energía. En un mundo con un acelerado crecimiento demográfico y por consiguiente, un rápido crecimiento de las necesidades, que ha provocado un estrés en los ecosistemas con su consecuente degradación. La creación del concepto de servicios ecosistémicos (SE) ha servido para realzar la interdependencia que tiene con los ecosistemas naturales (Boyd \& Banzhaf, 2007; Costanza et al., 2007; Durán, Casalegno, Marquet, \& Gaston, 2013; Gómez-Baggethun, de Groot, Lomas, \& Montes, 2010; Lara et al., 2009; Villa et al., 2014). El Millennium Ecosystem Assessment (MA, 2005), define a los SE como los beneficios que las personas obtienen de los ecosistemas.

La existencia de las áreas protegidas facilita la conservación de varios ecosistemas, entre ellos los bosques. En este contexto, van Beukering, Cesar, \& Janssen (2003) estudiaron los beneficios que entrega el Parque Nacional Leuser en Indonesia, y analizaron tres posibles escenarios, los cuales eran deforestación, conservación y un uso selectivo. Los resultados de este estudio muestran que la opción más beneficiosa económicamente era la de conservación (US\$9.538 millones), seguido por el uso selectivo (US\$9.100 millones) y la deforestación (US\$6.958 millones). Otro estudio, Portela \& Rademacher (2001), determina que en la Amazonia Brasileña los $\mathrm{SE}$ de las áreas protegidas proveen beneficios a nivel nacional y local de más del 50\% en comparación a la agricultura de pequeña escala. Adicionalmente, Amend, Reid, \& Gascón (2006) determinó, para la misma área de estudio, que la protección de esta área genera tres veces más beneficios económicos en comparación a la ganadería extensiva, que es el uso alternativo más probable para este parque.

Los bosques contribuyen con diversos beneficios para las personas, la gran biodiversidad que entregan es aprovechada por los seres humanos y generan múltiples SE, dentro de ellos se pueden encontrar protección de cuencas, ciclo de nutrientes, provisión de alimentos, recreación, polinización, regulación del clima local y global, entre otros.

A pesar de esto, se ha demostrado que en el último tiempo este tipo de ecosistema ha ido reduciéndose paulatinamente, ya sea por el cambio de uso de suelo a áreas agrícolas como también a plantaciones forestales de especies exóticas. De acuerdo al MA (2005), el área global de los sistemas forestales se ha reducido a la mitad en los últimos tres siglos, donde los bosques efectivamente han desaparecido en 25 países y en otros 29 han perdido alrededor del $90 \%$ de su cobertura forestal.

De acuerdo a este estudio se destacan dos servicios, el aprovisionamiento de productos forestales no maderable (PFNM) y el servicio cultural de recreación. Según la Organización de la Naciones Unidas para la Alimentación y la Agricultura (FAO), los PFNM se definen como "los bienes de origen biológico, diferentes a la madera, procedentes de los bosques, de otras tierras boscosas y de árboles fuera de los bosques". Es así que en esta clasificación se pueden encontrar diversos productos como aditivos alimentarios y los alimentos señalando frutos secos comestibles, setas, frutas, hierbas, especias y condimentos, plantasaromáticas, fibras utilizados en la construcción, muebles, ropa o utensilios, resinas, gomas y plantas. 
Muchas comunidades alrededor del mundo aprovechan estos productos y los ayudan ya sea para su subsistencia o como medio de ingresos. De acuerdo a los datos proporcionados por FAO para 2003, alrededor del $80 \%$ de la población en vías de desarrollo usan los PFNM por necesidades de salud o nutricionales.

En Chile se identifican alrededor de 350 PFNM (Chung, Sotomayor, \& Lucero, 2011) que son utilizados como sustento para proveer ingresos a las comunidades. La gama de PFNM en Chile es bastante variada, un poco menores en cantidad que en las zonas tropicales pero son de gran importancia, porelaltogradodeendemismo existente y por la gran variabilidad de condiciones edafoclimáticas que dependen del tipo de suelo, clima y altura en las que se presentan. Estimándose que alrededor de 220.000 personas participan en Chile en la recolección de PFNM, dando trabajo en zonas rurales principalmente a mujeres y niños (Valdebenito, 2012).

Si bien los PFNM son importantes en varias comunidades y otorgan un sinfín de beneficios, debido a su gran variabilidad, su producción es irregular y heterogénea. Además a esto, la información existente es casi nula, por lo que hoy en día no se toman en cuenta en las estadísticas nacionales ni tampoco en las políticas públicas ni estrategias de desarrollo.

El SE de recreación se puede definir como el placer recreacional de las personas derivado de los ecosistemas naturales o cultivados (MA, 2005). Es un SE cultural de uso directo. Las personas usualmente eligen pasar su tiempo de ocio en ecosistemas con características específicas. Las áreas protegidas entregan paisajes y escenarios naturales, a los cuales las personas tienden a concurrir para admirar y disfrutar el tiempo en estoslugares.
La cultura humana está fuertemente influenciada por los ecosistemas, por lo que algún cambio en ellos significa un impacto en la identidad cultural y la estabilidad social. Los cambios en los servicios culturales pueden tener una fuerte influencia en la salud, ya que afectan las oportunidades espirituales, de inspiración, estéticas y recreativas y estos a su vez afectar tanto los estados físicos y emocionales de las personas.

El uso de los servicios culturales han ido en aumento, pero la capacidad de los ecosistemas de proveer beneficios culturales ha ido disminuyendo significativamente en el último siglo. Las culturas humanas, los sistemas de conocimiento, religiones, valores patrimoniales, interacciones sociales y los servicios de esparcimiento (como son el disfrute estético, recreación, la realización artística y espiritual y el desarrollo intelectual) siempre han sido influenciados por las formas que provee los ecosistemas naturales y las condiciones que se encuentran. Muchos de estos beneficios se están degradando, ya sea a través de cambios en los ecosistemas o por medio de cambios en la sociedad que reduce el reconocimiento o aprecio de la gente de esos beneficios culturales. La rápida pérdida de ecosistemas y paisajes de valor cultural puede contribuir a trastornos sociales y la marginación social. Y además ha tenido una disminución en la cantidad y calidad de los paisajes naturales estéticamente agradables. Sin embargo, dentro de los servicios culturales, los SE de recreación y turismo están generalmente en buenas condiciones y en crecimiento, pero el mal manejo de las actividades turísticas y recreativas pueden eventualmente reducir la capacidad que tienen los ecosistemas de proveer estos servicios (MA, 2005).

La necesidad de llevar a cabo una buena gestión de los espacios naturales obliga a 
tener un conocimiento adecuado e integrado sobre las relaciones que se presentan entre los elementos naturales y humanos. Es así como una buena caracterización y cuantificación de los SE puede ayudar a un buen manejo de los mismos. Los SE dependen netamente de la interacción de los sistemas humanos y los ecosistemas por lo que la caracterización de un SE no solo se ve en ámbitos ecológicos, sino que también se debe lograr una caracterización socioeconómica del SE aprovechado. Una vez que se conoce qué servicios pueden ser suministrados por el ecosistema y cuáles son los servicios demandados por el sistema social, beneficiarios, se puede evaluar cuál es el flujo de los SE que se da entre ecosistema y el sistema socio-económico.

Para ello, se realiza una sectorización ecológica, la cual consiste en tipificar los ecosistemas, es decir, diferenciar sectores ambientales en base a su homogeneidad. También se debe generar una identificación del espacio que ocupan, la estructura, composición y funcionamiento de los ecosistemas. Pero además se debe evaluar el estado de los ecosistemas en el área de estudio, la descripción del proceso biofísico que determina la existencia de los SE que se estudian y la vinculación de su mantenimiento o pérdida con las actividades económicas.

Por otro lado, se debe generar una sectorización socio-económica en base a ciertas variables las cuales identificarán comunidades homogéneas. Los beneficiarios, son definidos como aquellos actores sociales que se benefician, directa o indirectamente, de los servicios suministrados por los ecosistemas; así como aquellas personas e instituciones que pueden verse afectadas positivamente por los modelos de gestión existentes, en cuanto a la mejora en el flujo de servicios (Martín-López \& Montes, 2010).

El de SE implica técnicas cartográficas no convencionales que integran herramientas de modelación espacialmente explícita de variable ecológicas con actividades de consulta ciudadana (mapeo participativo). Uno de los objetivos centrales del mapeo de $\mathrm{SE}$ es establecer la congruencia espacial entre la oferta de uno o más SE y la demanda que las comunidades ejerce sobre dichos SE. Esto permite orientar la toma de decisiones respeto al uso del territorio y la conservación de ecosistemas claves en la provisión SE (Haines-Young \& Potschin, 2010).

La noción de las dimensiones espaciales de los SE ayuda a visualizar los flujos y las cantidades de estos servicios dentro del área de estudio, es así como se convierte en una útil herramienta en la toma de decisiones y en el manejo de estos SE.

Nahuelhual, Carmona, Lozada, Jaramillo, \& Aguayo, 2013; Nahuelhual, Carmona, Laterra, Barrena, \& Aguayo, 2014, señalan que mapear un SE como es la recreación y el ecoturismo puede servir para múltiples propósitos desde diseñar hotspots de recreación cercanos a destinar recursos para nuevas iniciativas ecoturísticas en áreas donde el potencial de recreación es conocidamente alto.

De esta forma los Sistemas de Información Geográfica (SIG) son útiles debido a que tienen la capacidad de incorporar las complejidades de las dimensiones espaciales involucradas. Son particularmente útiles en regiones montañosas, donde la compleja topografía $\mathrm{y}$ las distintas gradientes ambientales requieren una especial consideración de los patrones espaciales (Grêt-Regamey, Bebi, Bishop, \& Schimd, 2008), esto ayuda en el entendimiento del comportamiento de los $\mathrm{SE}$ en la región dando más detalles de la distribución espacial de un ecosistema, los administradores y tomadores de decisiones puedan identificar fácilmente donde es apto para la explotación de recursos y donde deben ser conservados, así como entender la relación positiva entre el desarrollo económico, la intervención del gobierno y la conservación de los ecosistemas (Chen, Li, \& Wang, 2009). 
Este estudio busca cuantificar el stock de la provisión de servicios ecosistémicos relacionados con el aprovisionamiento de PFNM y recreación según el grado de naturalidad de las formaciones boscosas del Área de Alto Valor de Conservación (AAVC) de Caramávida.

\section{Materiales y métodos}

\section{Área de estudio}

El área de estudio es el AAVC de Caramávida, este se emplaza en la Provincia de Arauco ( $37^{\circ} 40^{\prime} 60 \mathrm{~S}, 73^{\circ}$ $20^{\prime} 60 \mathrm{~W}$ ) y comprende una superficie de 21.960 ha. Se destaca por ser un sitio prioritario para la biodiversidad y se encuentra supervisado por Forestal Arauco S.A. Para efectos de este estudio, se trabajó con el Área de influencia ecológica (figura 1). La definición de esta área de influencia ecológica se basó en la combinación de tres criterios generales, las cuales se determinaron para el plan de manejo de esta AAVC, los cuales son los siguientes:

Presencia de subcuencas hidrográficas cuya parte superior se encuentra dentro o en las inmediaciones de los límites del AAVC y cuya protección tiene directa relación con la cantidad y calidad de agua que en ellas se produzca.

Presencia de espacios vitales, tales como formaciones vegetacionales nativas $y$ cuerpos de agua, que conforman hábitat específicos de especies de interés para la conservación y que le dan continuidad a los procesos ecosistémicos que se desarrollan dentro del área protegida.

Establecimientodecorredoresbiológicosque permiten la interconexión, el mantenimiento y la dispersión de flujos genéticos entre poblaciones de flora y fauna, con otras áreas protegidas cercanas. Para el área de estudio en particular, se consideró el Parque Nacional Nahuelbuta y Trongol.

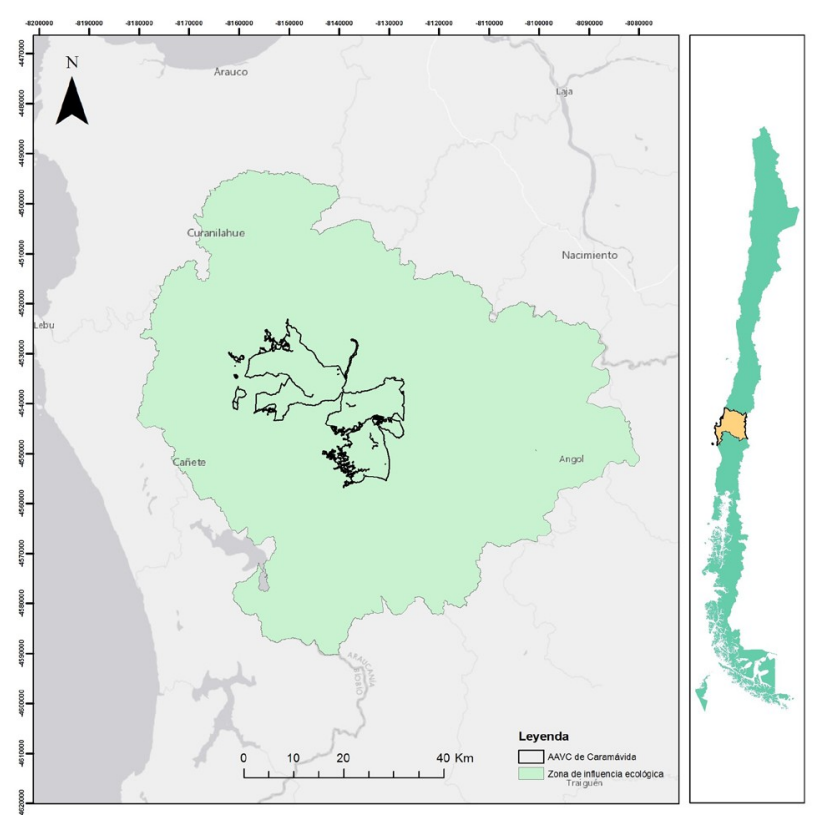

Figura 1. Ubicación del AAVC de Caramávida. Fuente: Elaboración propia (2018)

Figure 1. Location of the High Conservation Area Value (HCVA) of Caramávida. Source: Own elaboration (2018) 


\section{Mapeo de formaciones boscosas según} grado de naturalidad.

De acuerdo a este punto se realizó una revisión del Plan de Manejo del AAVC Caramávida, que fue generado con el objetivo de conservación de los ecosistemas de este sector, además de la definición del concepto de grado de naturalidad. Posteriormente se realizó la cartografía en el programa software ArcGis 10.1.

\section{Metodología para productos forestales no maderables.}

Para la caracterización de los PFNM en primera instancia se determinó qué productos son los que ofrece el sector Caramávida, para ello se realizó una revisión bibliográfica. En el Plan demanejo del AAVC Caramávida existe un registro de las especies que se encuentran en el sector de Caramávida, la cual se relacionó con el Catastro de PFNM en Bosque Nativo de la Corporación Nacional Forestal (CONAF) e Instituto Forestal (INFOR), así se logró obtener una tabla de todas las especies que pueden generar productos forestales no maderables y los usos que se le pueden dar a estos. Además se procedió a describir los PFNM más extraídos de acuerdo a la literatura.

Posteriormente se construyó una matriz cuyas columnas están indicados los PFNM identificados para el área de estudio y en las filas se indican las formaciones boscosas identificadas, de esta forma se determinó por cada formación boscosa la presencia $\mathrm{y}$ no presencia de los PFNM. A partir de esto, se generó la cartografía a través del programa software ArcGIS 10.1 con la información recopilada, presentando la distribución espacial de cantidad de las especies de PFNM en las diferentes formaciones boscosas.

\section{Metodología para servicio cultural de recreación.}

Para el caso del servicio de recreación se utilizó la metodología propuesta por Nahuelhual et al. (2013), la cual se resume en cinco pasos que se mencionan a continuación:

- Selección de los atributos y los criterios espaciales que determinan la recreación y ecoturismo.

- Validación de los atributos y criterios espaciales por medios de técnicas participativas.

- Análisis espacial y construcción del criterio espacial final.

- Ponderando los criterios espaciales utilizando el proceso analítico jerárquico.

- Corrección del REPf para los criterios de sostenibilidad para obtener el indicador final de oportunidades de la recreación y ecoturismo REOi.

\section{Resultados}

\section{Mapeo de formaciones boscosas según grado de naturalidad}

En relación a las formaciones boscosas, estas se definieron de acuerdo al Plan de Manejo de AAVC de Caramávida en conjunto con la Clasificación de pisos de vegetación y análisis de representatividad ecológica de áreas propuestas para la conservación en la eco-región (Luebert \& Pliscoff, 2006), en el cual se seleccionaron los siguientes pisos vegetacionales, en relación a la presencia en el área estudiada:

Bosque caducifolio transicional andino de Nothofagus alpina y Nothofagus obliqua. Bosque caducifolio templado andino de 
Nothofagus alpina y Nothofagus dombeyi. Bosque mixto templado de Nahuelbuta de Nothofagus dombeyi y Nothofagus obliqua. Bosque laurifolio templado costero de Aextoxicon punctatum y Laurelia sempervirens.

Bosque caducifolio transicional interior de Nothofagus obliqua y Cryptocarya alba.

Bosque caducifolio templado de Nothofagus obliqua y Persea lingue.

Bosque resinoso templado de Nahuelbuta de Araucaria araucana.

Matorral caducifolio templado andino de Nothofagus antarctica y Empetrum rubrum.

A partir de estas formaciones boscosas, se definió el grado de naturalidad de acuerdo al documento Método para la Planificación del Manejo de Áreas Protegidas (Núñez, 2010), por lo que el grado de naturalidad, se puede definir como el grado de independencia de actuaciones humanas en una comunidad vegetal, o la escasa intervención o acción transformadora del ser humano, representado en el estado y composición de la flora autóctona libre de intervención antrópica. A partir de esta definición, se especifican distintos grados de naturalidad los cuales se resumen en la tabla 1 .

Concorde a lo establecido en el Método para la Planificación del Manejo de Áreas Protegidas y de acuerdo a la información recopilada por el Plan de manejo delAAVC de Caramávida, se logra obtener la figura 2, en la cual se muestra las diferentes formaciones boscosas dentro del área de estudio, mientras que la figura 3 muestra el grado de naturalidad para toda el área de influencia.

Tabla 1

Grado de naturalidad. Fuente: CONAF, Método para la planificación del manejo de áreas protegidas (2008)

Table 1

Degree of naturalness. Source: CONAF, Guidelines for the management planning of protected areas (2008)

\begin{tabular}{lcl}
\hline Calificación & Valor & Descripción \\
\hline Nula & 1 & $\begin{array}{l}\text { Flora en gran medida exótica (plantaciones o introducidas); } \\
\text { sin estructura vegetal natural o enteramente destruida; sectores } \\
\text { altamente antropizados }\end{array}$ \\
Muy Baja & 20 & $\begin{array}{l}\text { Flora autóctona (puede ser empobrecida) mezclada con exóticas } \\
\text { (pueden ser plantaciones); estructura vegetal natural muy } \\
\text { modificada y artificial; actividades intensivas, permanentes o } \\
\text { esporádicas (explotación forestal, pastoreo, otros) }\end{array}$ \\
Baja & 40 & $\begin{array}{l}\text { Flora autóctona muy empobrecida; estructura vegetal natural muy } \\
\text { modificada; actividades intensivas, permanentes o esporádicas } \\
\text { (pastoreo, explotación forestal) }\end{array}$ \\
Media & 60 & $\begin{array}{l}\text { Flora autóctona empobrecida; estructura vegetal natural } \\
\text { modificada; actividades extensivas, actual o abandonadas } \\
\text { (pastoreo, recolección frutos, otros), a menudo de tipo ancestral } \\
\text { Flora autóctona modificada; estructura vegetal natural con algún } \\
\text { grado de simplificación; nula o escasa intervención humana }\end{array}$ \\
Alta & 80 & $\begin{array}{l}\text { Flora autóctona inicial; estructura vegetal natural compleja o } \\
\text { poco simplificada (comunidades climácicas o permanentes); nula } \\
\text { intervención humana. }\end{array}$ \\
\hline
\end{tabular}




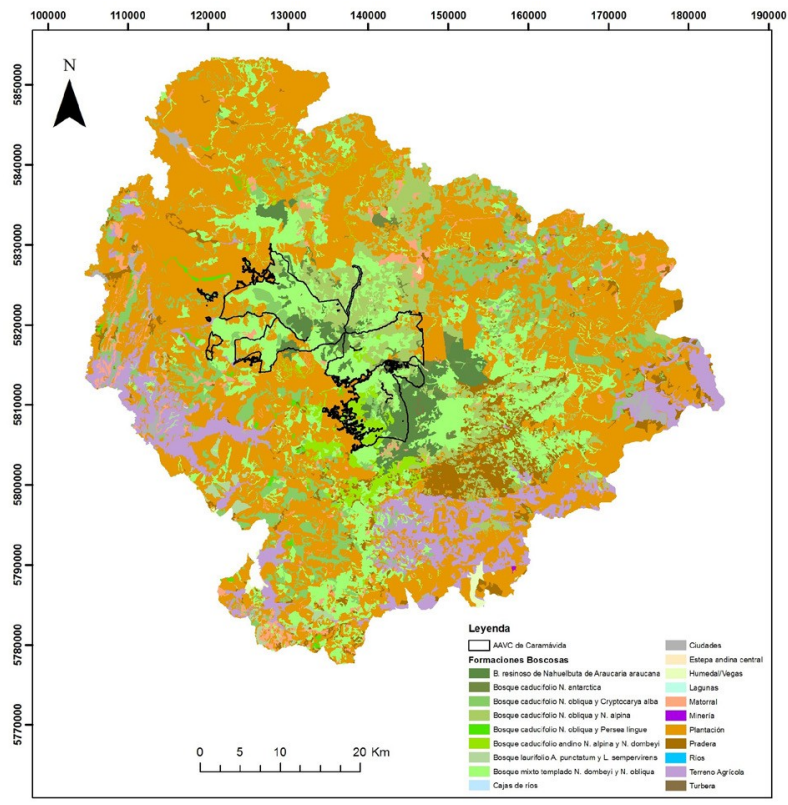

Figura 2. Formaciones boscosas del área de influencia ecológica. Fuente: Elaboración propia (2018)

Figure 2. Forest formation in the area of ecological influence. Source: Own elaboration (2018)

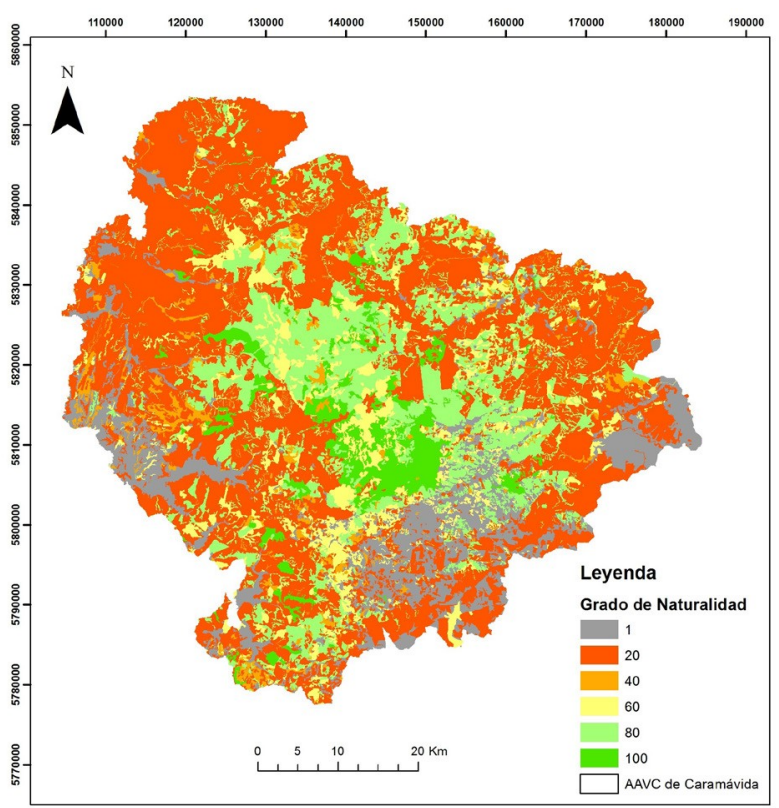

Figura 3. Grado de naturalidad del área de influencia ecológica. Fuente: Elaboración propia (2018)

Figure 3. Degree of naturalness in the area of ecological influence. Source: Own elaboration (2018)

A raíz de esto, se pudo obtener la tabla

2 , donde muestra la ocupación de cada formación boscosa, según grado de naturalidad. 
Tabla 2

Ocupación de las formaciones boscosas. Fuente: Elaboración propia (2018)

Table 2

Use of the forest formations. Source: Own elaboration (2018)

\begin{tabular}{|c|c|c|c|c|}
\hline Formación boscosa & Naturalidad & $\begin{array}{r}\text { Superficie } \\
\left(\mathrm{km}^{2}\right)\end{array}$ & $\%$ de superficie & Total $\left(\mathrm{km}^{2}\right)$ \\
\hline \multirow{6}{*}{$\begin{array}{l}\text { Bosque resinoso de } \\
\text { Nahuelbuta de Araucaria } \\
\text { araucana }\end{array}$} & 100 & 39,61 & 36,53 & \multirow{6}{*}{108,44} \\
\hline & 80 & 41,38 & 38,16 & \\
\hline & 60 & 20,65 & 19,04 & \\
\hline & 40 & 6,79 & 6,26 & \\
\hline & 20 & 0,01 & 0,01 & \\
\hline & 1 & 0,00 & 0,00 & \\
\hline \multirow{6}{*}{$\begin{array}{l}\text { Bosque caducifolio } \\
\text { Nothofagus antarctica }\end{array}$} & 100 & 14,16 & 57,62 & \multirow{6}{*}{24,57} \\
\hline & 80 & 3,86 & 15,73 & \\
\hline & 60 & 3,13 & 12,72 & \\
\hline & 40 & 1,91 & 7,78 & \\
\hline & 20 & 1,51 & 6,15 & \\
\hline & 1 & 0,00 & 0,00 & \\
\hline \multirow{6}{*}{$\begin{array}{l}\text { Bosque caducifolio } \\
\text { Nothofagus obliqua y } \\
\text { Cryptocarya alba }\end{array}$} & 100 & 21,41 & 8,56 & \multirow{6}{*}{250,19} \\
\hline & 80 & 83,64 & 33,43 & \\
\hline & 60 & 95,36 & 38,12 & \\
\hline & 40 & 37,19 & 14,86 & \\
\hline & 20 & 12,58 & 5,03 & \\
\hline & 1 & 0,00 & 0,00 & \\
\hline \multirow{6}{*}{$\begin{array}{l}\text { Bosque caducifolio } \\
\text { Nothofagus obliqua y } \\
\text { Nothofagus alpina }\end{array}$} & 100 & 7,03 & 2,72 & \multirow{6}{*}{258,95} \\
\hline & 80 & 167,96 & 64,86 & \\
\hline & 60 & 77,16 & 29,80 & \\
\hline & 40 & 6,05 & 2,34 & \\
\hline & 20 & 0,75 & 0,29 & \\
\hline & 1 & 0,00 & 0,00 & \\
\hline \multirow{6}{*}{$\begin{array}{l}\text { Bosque caducifolio } \\
\text { Nothofagus obliqua y } \\
\text { Persea lingue }\end{array}$} & 100 & 0,00 & 0,00 & \multirow{6}{*}{10,85} \\
\hline & 80 & 1,96 & 18,06 & \\
\hline & 60 & 2,92 & 26,89 & \\
\hline & 40 & 5,23 & 48,17 & \\
\hline & 20 & 0,75 & 6,88 & \\
\hline & 1 & 0,00 & 0,00 & \\
\hline
\end{tabular}




\begin{tabular}{lrrrr}
\hline Formación boscosa & Naturalidad & $\begin{array}{r}\text { Superficie } \\
\left(\mathrm{km}^{2}\right)\end{array}$ & \% de superficie & Total $\left(\mathrm{km}^{2}\right)$ \\
\hline & 100 & 14,81 & 22,86 & \\
Bosque caducifolio andino & 80 & 25,20 & 38,92 & \\
Nothofagus alpina y & 60 & 24,75 & 38,22 & \\
Nothofagus dombeyi & 40 & 0,00 & 0,00 & 64,76 \\
& 20 & 0,00 & 0,00 & \\
& 1 & 0,00 & 0,00 & \\
& & & & \\
Bosque laurifolio & 100 & 3,15 & 13,25 \\
Aextoxicon punctatum y & 80 & 10,63 & 44,78 \\
Laurelia sempervirens & 60 & 7,13 & 30,03 & \\
& 40 & 2,84 & 11,94 & \\
& 20 & 0,00 & 0,00 & \\
& 1 & 0,00 & 0,00 & \\
Bosque mixto templado & & & & \\
Nothofagus dombeyi y & 100 & 88,91 & 16,99 & \\
Nothofagus obliqua & 80 & 265,00 & 50,63 & \\
& 60 & 127,71 & 24,40 & \multirow{2}{*}{523,43} \\
& 40 & 33,58 & 6,42 & \\
& 20 & 8,24 & 1,57 & \\
& 1 & 0,00 & 0,00 & \\
& & &
\end{tabular}

Servicio de aprovisionamiento de PFNM.

Dentro de la caracterización de los PFNM del área de influencia se encontraron variadas especies que entregan estos productos, entre ellos árboles, arbustos, hongos, helechos y trepadoras, las cuales tienen distintos usos y algunas pueden utilizarse para diferentes usos. En el área de estudio la principal actividad gira en torno a la recolección de piñones, murta, hongos como changle, digüeñes y loyo, y algunas plantas medicinales, sin embargo, el estudio no se centró en estas últimas, ya que son especies muy variadas y difíciles de caracterizar y cuantificar. Es por esto, que el estudio se enfocó en la recolección de frutos y hongos.

Piñón: La especie Araucaria araucana produce frutos comestibles en forma natural y silvestre. Las flores femeninas tienen forma de conos en espiral y se desarrollan en los extremos de ramas, solas o de a dos. Las flores femeninas también sedesarrollan en el extremo de ramillas cortas, en forma conos alargados. El fruto es la semilla que se denomina piñón y su formación completa demora 16 a 18 meses, desde la fertilización. Un árbol puede producir entre 20 a 40 conos por árbol y cada cono frutal puede contener un promedio de 100 a 200 semillas o piñones. Los piñones son comestibles y formaban parte importante de la dieta de los pueblos originarios, debido a su alto contenido energético y que actualmente aún se siguen recolectando y comercializando, de acuerdo a lo mencionado por la Fundación para la Innovación Agraria del Ministerio de Agricultura. En la figura 4 se aprecia la distribución espacial de la presencia y ausencia de la especie Araucaria Araucana, las zonas anaranjadas son aquellas que indican la presencia de esta especie alcanzando un área de $108,4 \mathrm{~km}^{2}$. 
Murta: La Murta o Murtilla (Ugni molinae), es una especie que pertenece a la familia de las Myrtacea, crece en Chile desde Talca hasta el Río Palena, encontrándose habitualmente en terrenos despejados, en los bordes del Bosque o formando parte del matorral arbustivo. La planta produce frutos comestibles, las que consisten en bayas globosas y pequeñas, de agradable sabor y aroma. Los usos que se le dan a esta planta son variados los frutos sirven de alimento y se utilizan en la elaboración de mermeladas, jarabes, postres y licores, también tiene uso medicinal, ya que posee propiedades aromáticas, es estimulante $\mathrm{y}$ astringente.

Otro uso es el Ornamental de la planta, debido a las características de sus flores blanco - rosadas y su follaje perenne es de color verde con visos rojizos, muy agradables a la vista tal como señalan varios especialistas en el tema. El fruto de la murta o murtilla es recolectado en zonas rurales, luego es transportado a centros urbanos, donde es comercializado a consumidores finales o intermediarios, que destinan dicho producto a otros mercados nacionales o internacionales. En la figura 4, se muestra la presencia de la especie Ugni molinae, murta, en la que se puede apreciar que en las zonas de color anaranjado se encuentra la posible presencia de esta especie y la zona azul representa la ausencia de esta, la cual crece principalmente en el Bosque mixto templado de Nahuelbuta de Nothofagus dombeyi y Nothofagus obliqua, representando una superficie de 523,4 $\mathrm{km}^{2}$.

Hongos: Aquí cabe mencionar que los principales hongos que se extraen en el área de influencia son el changle (Ramaria flava), digüeñes (Cyttaria espinosae) y loyo (Boletus loyo). A continuación se describen cada uno de estos:
Changle (Ramaria flava): Es un hongo con forma de coral. El cuerpo fructífero es varias veces ramificado hacia las puntas, de color amarillo azufre, tornándose blanco hacia la base del estípite. Comúnmente, las ramificaciones terminan en una doble punta, con una bifurcación en forma de $\mathrm{U}$ o V.El uso que se le da es comestible y se caracteriza por poseer un olor suave y un delicado sabor. Se le puede encontrar en el interior de los bosques en zonas húmedas $\mathrm{y}$ oscuras.

Digüeñes (Cyttaria espinosae): Es un parásito que se caracteriza por sus fructiferaciones de color blanco intenso, aunque es posible encontrar algunos individuos más amarillentos. Es de forma globosa con un pequeño estípite. Una delgada membrana blanca lo cubre y se rompe al crecer, descubriendo orificios penta o hexagonales. Es de contextura firme, elástica y tiene una textura pegajosa. No posee olor característico, pero sí un sabor dulzón yuna textura chiclosa al cocinarla.

Loyo (Boletus loyo): Esta fructiferación del famoso género Boletus, muchos de los cuales son comestibles, es uno de los pocos encontrados en Chile. La enorme fructiferación con el sombrero de color rojoburdeo, de forma redondeada cuando joven y convexo al envejecer con una textura lisa y seca. La cara inferior del sombrero, que está cubierto de tubos, es de color amarillo intenso cuando inmaduro, tornándose verde con tintes azules al envejecer. Es de textura fibrilosa y de contextura gruesa y firme. Tiene un olor fúngico suave y un sabor agradable. Se consume fresco y seco. En su época de aparición se vende muy rápidamente, pues tiene alto valor gastronómico. Se encuentra al interior de los bosques en zonas húmedas y oscuras, cerca de "hualles", roble joven (Furci George-Nascimiento, 2007). 
Todos estos hongos crecen en similares condiciones por lo que se pueden encontrar en las mismas formaciones boscosas como Bosque caducifolio de Nothofagus alpina y Nothofagus obliqua, Bosque caducifolio de Nothofagus alpina y Nothofagus dombeyi, Bosque mixto templado de Nahuelbuta de Nothofagus dombeyi y Nothofagus obliqua, Bosque caducifolio de Nothofagus obliqua y Cryptocarya alba,
Bosque caducifolio de Nothofagus obliqua y Persea lingue y finalmente en el Bosque caducifolio Nothofagus Antarctica. En la figura 4 se aprecia la posible presencia o ausencia de estas especies, dentro de la zona de influencia, las áreas anaranjadas son aquellas donde posiblemente crezcan estos hongos, que son las áreas que ocupan las formaciones boscosas mencionadas anteriormente.
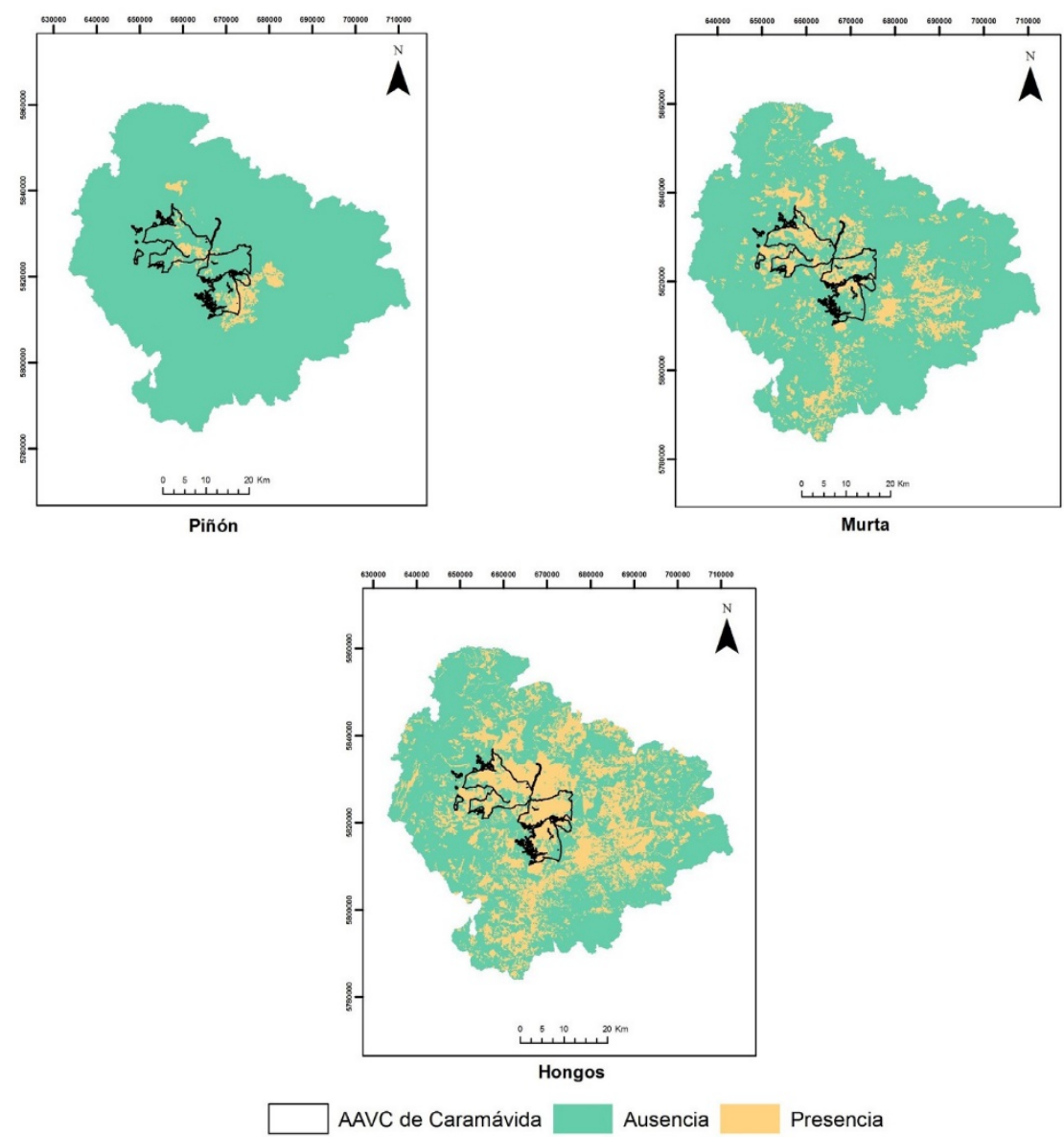

Figura 4. Ubicación de especies de PFNM más extraídas. Fuente: Elaboración propia (2018)

Figure 4. Location of the most frequently extracted species of non-timbr forest Products. Source: Own elaboration (2018)

Secuantificólacantidadpotencialde especies que generan PFNM. En primer lugar, se identificó a través de una matriz la presencia o ausencia de cada especie de PFNM en cada formación boscosa según literatura, lo que dio resultado a una matriz que está resumida en la tabla 3. La distribución espacial de estas especies se aprecia en la figura 5. 
Tabla 3

Número de especies de PFNM por formación boscosa. Fuente: Elaboración propia (2018)

Table 3

Number of species of non-timber forest products per forest formation. Source: Own elaboration (2018)

Formación Boscosa

$\mathrm{n}^{\circ}$ de especies

PFNM

Bosque caducifolio transicional andino de Nothofagus alpina y N. obliqua

32

Bosque caducifolio templado andino de Nothofagus alpina y N. dombeyi

Bosque mixto templado de Nahuelbuta de Nothofagus dombeyi y $N$.

obliqua

Bosque laurifolio templado costero de Aextoxicon punctatum y Laurelia sempervirens

Bosque caducifolio transicional interior de Nothofagus obliqua y

Cryptocarya alba

Bosque caducifolio templado de Nothofagus obliqua y Persea lingue

Bosque resinoso templado de Nahuelbuta de Araucaria araucana

Matorral caducifolio templado andino de Nothofagus antarctica y

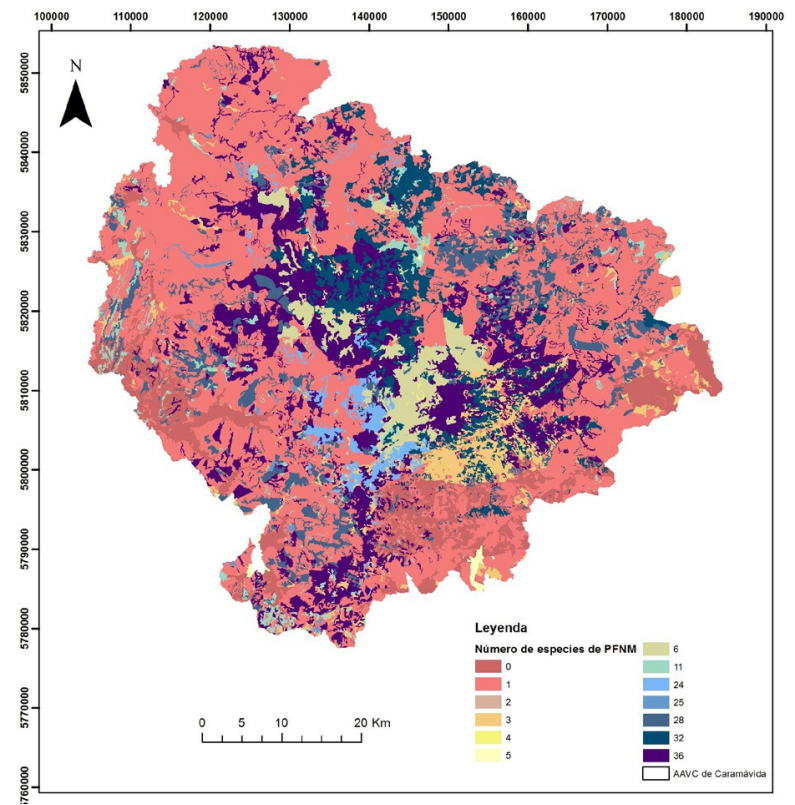

Figura 5. Distribución de especies de PFNM en el área de influencia. Fuente: Elaboración propia (2018)

Figure 5. Distribution of species of non-timber forest products in the area of interest. Source: Own elaboration (2018) 


\section{Servicio cultural de recreación}

Como ya se mencionó, para caracterizar y cuantificar se utilizó la metodología propuesta por Nahuelhual et al. (2013):

Selección de los atributos y los criterios espaciales que determinan la recreación y ecoturismo. Para el AAVC se seleccionó atributos de acuerdo a literatura, en donde se muestra las variables y escalas de medición que se sometieron a validación por expertos (tabla 4).

Validación de los atributos y criterios espaciales por medios de técnicas participativas. En este punto se realizaron entrevistas a 11 personas expertas en el tema, que se dedicaran al rubro del turismo y que conocieran a profundidad el área de estudio.

Análisis espacial y construcción del criterio espacial final. De acuerdo a las entrevistas realizadas a los expertos se obtuvo la validación de la mayoría de los criterios, estableciendo los expuestos en la tabla 4.

Ponderando los criterios espaciales utilizando el proceso analítico jerárquico. En este punto se logró obtener la ecuación de prioridad final, la cual se puede resumir en la siguiente expresión:

$R E P f=0,36 * R N S+0,14 * A+0,38 * B E+0,15 * C A T$

Con esta ecuación se mapeo para obtener una función del Potencial de Recreación y Ecoturismo (REPf), que se resume en la figura 6 .

\section{Discusión}

\section{Mapeo de formaciones boscosas}

A partir de la figura 2 y figura 3 , se puede destacar que las plantaciones forestales ocupan gran parte del territorio, con un muy bajo grado de naturalidad. También existen otros usos que tienen un bajo grado de

\section{Tabla 4}

Atributos espaciales y escala de medición elegidos. Fuente: Elaboración propia (2018)

Table 4

Spatial attributes and the scale of chosen measurement. Source: Own elaboration (2018

\begin{tabular}{llll} 
Dimensión & Atributo & Variable de medición & $\begin{array}{l}\text { Escala de } \\
\text { medición }\end{array}$ \\
\hline \multirow{2}{*}{ Ecosistema } & $\begin{array}{l}\text { Recursos Naturales } \\
\text { Singulares }\end{array}$ & $\begin{array}{l}\text { Alto de los bosques nativos } \\
\text { Presencia de cuerpos de agua } \\
\text { Grado de naturalidad }\end{array}$ & $0-100$ \\
& & Cuenca visual & $0-100$ \\
Paisaje & Belleza escénica & Concentración de caminos secundarios & $0-100$ \\
Territorio & Accesibilidad & Concentración de atractivos naturales & $0-100$ \\
& Aptitud para el uso & Variedad de atracciones turísticas & $0-100$ \\
\hline
\end{tabular}




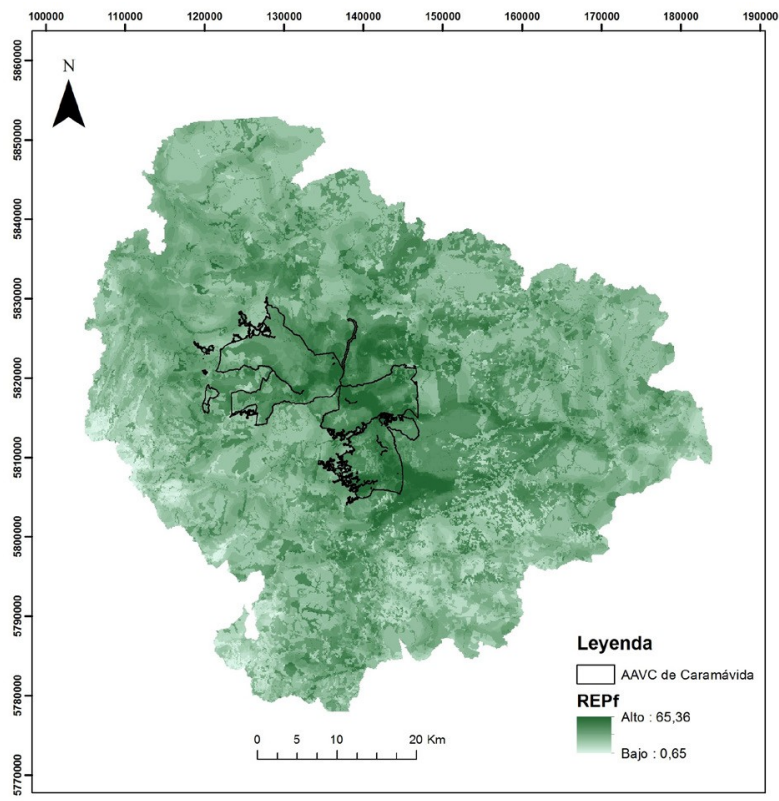

Figura 6. Valor de REPf para el área de estudio. Fuente: Elaboración propia (2018)

Figure 6. Value of the potential for recreation and ecotourism. Source: Own elaboration (2018)

naturalidad como son las ciudades, praderas y cultivos agrícolas, evidentemente son estos usos los que más intervención antrópica, aun así, existen formaciones boscosas que también constan de un bajo grado de naturalidad debido a la cercanía que tienen con estos usos y, que poseen un alto nivel de intervención. Por otro lado, las zonas con mayor grado de naturalidad se concentran tanto en el AAVC de Caramávida como alrededores, como es el Parque Nacional Nahuelbuta, que son los lugares en donde se encuentran la mayoría de las formaciones boscosas nativas y en general presentan un buen estado de conservación manteniendo un grado de naturalidad entre 60 a 100, es decir entre un grado medio a muy alto, esto es por el interés de conservación de estos bosques que han conllevado a tener recursos que ayudan en su protección.

Las formaciones boscosas que se encuentran dentro del AAVC de Caramávida y del
Parque Nacional Nahuelbuta principalmente tienen un grado de naturalidad que varía entre medio a alto (figura 2 y figura 3 ), con solo pequeños sectores con un grado bajo que son aquellos cercanos a caminos principales y aledaños a plantaciones y praderas donde existeactividadganadera. Además, seaprecia que fuera de estas zonas las formaciones boscosas están más fragmentadas y con un mayor grado de intervención, por ende menor grado denaturalidad.

La tabla 2 muestra la ocupación que tiene cada formación boscosa dentro del área de estudio, medida en $\mathrm{km}^{2}$, como también la ocupación que tiene de acuerdo al grado de naturalidad y su respectivo porcentaje. El Bosque mixto templado Nothofagus dombeyi y Nothofagus obliqua es el que representa una mayor extensión alcanzando $523,43 \mathrm{~km}^{2}$ y en su mayoría presenta un buen estado de conservación, ya que más de la mitad de su ocupación se encuentra en un 
grado alto de naturalidad. Contrariamente, la formación boscosa con menor extensión es el Bosque caducifolio Nothofagus obliqua y Persea lingue el cual solo ocupa $10,85 \mathrm{~km}^{2}$ del territorio estudiado y, que casi el $50 \%$ de este presenta un bajo grado de naturalidad, esto se debe al alto valor maderero de estas especies, las cuales han sido ampliamente explotadas, además que ha sido deforestada para la actividad agrícola y ganadera.

Bosque laurifolio Aextoxicon punctatum y Laurelia sempervirens, Bosque caducifolio Nothofagus antarctica y el Bosque caducifolio andino Nothofagus alpina y Nothofagus dombeyi también son formaciones boscosas que ocupan bajo territorio $\left(23,75 \mathrm{~km}^{2}, 24,57 \mathrm{~km}^{2}\right.$ y $64,76 \mathrm{~km}^{2}$ respectivamente), destacando esta última debido a que presenta buenas condiciones de conservación cuyo grado de naturalidad varía entre medio a alto, sin encontrarse extensiones con bajo grado de naturalidad.

Por otra parte, el Bosque caducifolio Nothofagus obliqua y Nothofagus alpina y el Bosque caducifolio Nothofagus obliqua y Cryptocarya alba también presentan una extensión amplia $\left(258,95 \mathrm{~km}^{2}\right.$ y $250,19 \mathrm{~km}^{2}$, respectivamente), pero el Bosque mixto templado Nothofagus dombeyi y Nothofagus obliqua sigue siendo significativamente mayor representado aproximadamente la extensión que ocupan ambos.

En general, ambas formaciones boscosas presentan más de la mitad de su extensión con un grado de naturalidad entre medio a alto, sin embargo, como se puede ver en la figura 3 el Bosque caducifolio Nothofagus obliqua y Cryptocarya alba se encuentra principalmentefueradeloslímitesdelAAVC de Caramávida, encontrándose en zonas más bajas y, está altamente fragmentado. Mientras que el Bosque caducifolio Nothofagus obliqua y Nothofagus alpina se encuentra más concentrado, tiene una mayor ocupación dentro del AAVC deCaramávida $\mathrm{y}$ alrededores, esto se debe que tienden a distribuirse sobre los $800 \mathrm{~m}$ de altura.

Finalmente, el Bosque resinoso de Nahuelbuta de Araucaria araucana tiene una extensión de 108,44 km² y se caracteriza por tener más de un $75 \%$ de su extensión en un grado de naturalidad alto a muy alto, esto es porque se desarrolla sobre los $1.000 \mathrm{~m}$ de altura y lugares de difícil acceso, además que la madera de esta especie y otras que están asociadas a esta, no tienen un alto valor maderero, por lo que han logrado mantener su naturalidad, sin embargo, esto no significa que la inadecuada recolección de PFNM puede afectar seriamente en la mantención de estos bosques. También cabe mencionar que esta especie se encuentra protegida y sus bosques dentro del área de estudio se encuentran fragmentados y están principalmente concentrados entre el Parque Nacional Nahuelbuta y el AAVC de Caramávida, encontrándose la mayoría de su ocupación dentro de estos territorios.

\section{Servicio de aprovisionamiento de PFNM.}

Como explica el Catastro de PFNM en Bosque Nativo de CONAF-INFOR, la escasa información relativa a los sistemas de producción, evaluaciones físicas cualitativas y cuantitativas, mercados, canales de comercialización, así como el desconocimiento de las empresas relacionadas con la comercialización y procesamiento de los PFNM por parte de los productores primarios o recolectores, repercute fuertemente en las posibilidades de desarrollo del PFNM en cuestión y por ende en los ingresos del pequeño propietario o recolector, que ve en esta actividad sólo una opción secundaria para mejorar sus ingresos $y$ no como una actividad económica diferente y que puede llegar a ser rentable. 
Piñón: En la figura 4, queda evidenciado que es un área bastante pequeña dentro del área de estudio y que se encuentra concentrada principalmente dentro de los límites del AAVC de Caramávida como en sitios colindantes, lo que quiere decir, que la recolección de piñones se concentra principalmente en estos sectores, por otra parte el área verde indica la ausencia de esta especie y se puede ver que la mayor parte del territorio no dispone de esta especie.

Murta: Como se ve en la imagen esta formación boscosa se encuentra bastante fragmentada, ocupando principalmente quebradas y se distribuye en casi toda la zona de influencia. Así mismo, se puede apreciar que dentro del AAVC de Caramávida se encuentran varias áreas donde se puede localizar esta especie.

Hongos: ocupan una superficie total de $1.132,7 \mathrm{~km}^{2}$, es decir, que existe unaamplia extensión de lugares donde se pueden encontrar estos productos, por el contrario las áreas en tonalidad verde indican la ausencia de estas formaciones boscosas, por lo tanto, es difícil encontrar estas especies en estos lugares. Además se puede ver como el bosque también se encuentra bastante fragmentado, sin embargo, está principalmente concentrado al centro del área de estudio, por lo que es visible que el AAVC de Caramávida posee gran proporción de lugares donde se pueden encontrar estas especies.

En general, existen muchas zonas donde se pueden recolectar especies de PFNM dentro del área de estudio como queda demostrado en la figura $5 \mathrm{y}$, en especial en el AAVC de Caramávida como alrededores, debido a que representan sus bosques un grado de naturalidad alto, la posibilidad de encontrar estas especies en buen estado es alta. Es así que el Bosque mixto templado de Nahuelbuta de Nothofagus dombeyi y Nothofagus obliqua y el Bosque laurifolio templado costero de Aextoxicon punctatum y Laurelia sempervirens, son las formaciones boscosas con mayor potencial en la recolección de PFNM, encontrándose en ellas hasta 36 especies que pueden ser aprovechadas, mientras la que menor número de especies que generan PFNM son Bosque resinoso templado de Nahuelbuta de Araucaria araucana y el Matorral caducifolio templado andino de Nothofagus antarctica y Empetrum rubrum, encontrándose solo 6 especies de PFNM.

En general la mayoría de las formaciones boscosas presentan un alto número de especies de PFNM, exceptuando las dos últimas mencionadas y por lo tanto, estas tienen un alto potencial de aprovechamiento de estos recursos. Los sectores con tonalidad azul a celeste indican un mayor número de especies encontradas para esos lugares, mientras que las tonalidades de amarillo a rosado representan un menor número de especies de PFNM, así queda demostrado que existen muchas zonas donde se pueden encontrar estos productos y que a pesar que en las formaciones boscosas se encuentran la mayoría de las especies, también se pueden encontrar en algunos usos como las plantaciones forestales, praderas, matorrales, humedales entre otros, pero en menor medida.

En los únicos usos que no encontróninguna especie de PFNM fue en las cajas de ríos, ciudades, en lugares donde se desarrolla la minería y en terrenos agrícolas. La zona en que más se concentran las especies de PFNM es al centro del área de estudio donde se ubica el AAVC de Caramávida, Parque Nacional Nahuelbuta y bosques nativos aledaños, las cuales son las zonas donde se concentra la actividad de recolección de PFNM.

En general, el bosque nativo presenta un número alto de especies que pueden ser aprovechadas para PFNM, encontrándose más de 20 especies que pueden ser aprovechadas con este fin, exceptuando el Bosque Resinoso Templado de Nahuelbuta de Araucaria araucana y el Matorral caducifolio templado andino de Nothofagus 
antarctica y Empetrum rubrum, dónde solo se hallaron 6 especies de PFNM.

También se puede apreciar que las zonas que tienen un alto grado de naturalidad es posible encontrar formaciones boscosas que brindan un alto número de especies PFNM, por lo que se puede deducir que estos dos factores están relacionados, es decir, si existe un cambio en el grado de naturalidad es probable que se vea afectado directamente el servicio de aprovisionamiento de PFNM.

\section{Servicio de recreación.}

La figura 6, muestra espacialmente los valores de REPf distribuidos por el área de estudio, de esta forma los valores más altos fueron de 65,36 que representa un valor entre medio a alto, mientras que el valor más bajo es de 0,65 , cuya calificación es más bien nula. Es decir, que las áreas de tonalidad verde oscuro representan un potencial recreativo alto, así mismo, las zonas de tonalidad verde claro a blanco representan una baja a nula potencialidad en recreación y ecoturismo.

También se puede decir que las áreas de más alto valor de potencial de recreación están dentro del AAVC de Caramávida, Parque Nacional de Nahuelbuta y alrededores de bosque nativo. Debido al interés de conservación de estos sectores, en estos se encuentran los valores más altos de los atributos espaciales anteriormente descritos, por ejemplo, se encontraron las cuencas visuales de más alto valor, también un mayor grado de naturalidad, así mismo, los bosques presentan mayor altura por ende una más alta valoración y así sucesivamente, de esta forma el valor de REPf es más alto para estas zonas.

Por otro lado, las áreas con menor potencial de recreación y ecoturismo son aquellas más intervenidas, como ciudades, praderas, terrenos agrícolas, entre otros y que están más alejadas de la quebrada de
Caramávida, que son zonas con menor valor de los atributos de belleza escénica, recursos naturales singulares y capacidad de atracción turística, ya que son zonas de mayor intervención y menos grado de naturalidad.

Para este estudio, no se pudo calcular el REOi, debido a la falta de información y de estudios del área, por lo que no se pudo calcular este indicador. Existe poco interés de parte de las instituciones en generar información de este tipo la zona, además de que existe información, pero que no está disponible para ser usada, lo que entorpece trabajos como éste.

\section{Conclusiones}

Primeramente, cabe mencionar que el stock o potencial está directamente relacionado con el grado de naturalidad, tanto del servicio de recreación como el de aprovisionamiento de productos forestales no maderables. En ambos, se observó que el potencial más alto, se concentraba en el AAVC de Caramávida, Parque Nacional Nahuelbuta y sectores aledaños, que son también las mismas zonas en los que predomina un grado de naturalidad de alto a muy alto y viceversa. Por esto, se puede decir que el grado de naturalidad está directamente relacionado con la provisión de estos servicios, así mismo, una degradación de las formaciones boscosas influiría directamente en la provisión del servicio de productos forestales no maderables como también en el servicio de recreación, es por esto que se puede decir que la conservación de las formaciones boscosas determina el stock o potencial de estos servicios. Por otro lado, a pesar que no se pudo calcularel flujo de ambos servicios debido a la falta de información, se podría pensar que ocurre lo mismo, es decir, que también depende del estado de conservación de las formaciones boscosas, ya que la disponibilidad de estos recursos estarían dadas, en gran medida, en aquellas zonas con mayor grado de naturalidad, por lo que su aprovechamiento también se daría en estas zonas. 
En relación a los PFNM se pudo calcular el stock o la potencialidad de extracción de estos productos, si bien, existen diversas especies que generan PFNM, comotambién existen diversos productos que pueden ser utilizados para diversos fines, esto en la actualidad, no se aprovecha en su totalidad, existe extracción de solo algunos recursos, principalmente algunas plantas para uso medicinal, digüeñes, changle, loyo, murta y piñones, los cuales se extraen en forma estacional y con creciente intensidad (Plan de Manejo del AAVC de Caramávida). Esta área tiene un alto potencial de estos productos que son desaprovechados por desconocimiento de las comunidades. En general todos los PFNM mencionados anteriormente se encontraron en el AAVC de Caramávida y existe un alto número de otras especies de PFNM que también que se encuentran en los como lugares donde habitualmente se extraen, por lo que se encontró un alto potencial de especies de PFNM dentro de los bosques del AAVC.

Dicho esto, queda en evidencia la necesidad de proteger estas áreas, el mantener niveles altos de naturalidad supone una conservación del alto potencial que existe de proveer estos servicios ecosistémicos, y de otros que no fueron incluidos en este estudio. Laextracción de estos recursos debe ser de manera sustentable, ya que la sobreexplotación puede generar un problema para la conservación del potencial que entregan los bosques en cuanto a servicios ecosistémicos.

\section{Referencias}

Amend M., Reid J., \& Gascón C. (2006). Benefícios econômicos locais de áreas protegidas na região de Manaus, Amazonas. Megadiversidade, 2(1-2). Recuperado de https://www.conservation-strategy.org/sites/ default/files/field-file/0_12AManaus_Parks_ Megadiversidade_beneficios.pdf

Boyd J. \& Banzhaf S. (2007). What are ecosystem services? The need for standardized environmental accounting units. Ecological economics, 63(2-3), 61662. https://doi.org/10.1016/j.ecolecon. 2007.01.002

Costanza, R., d'Arge, R., de Groot, R.S., Farber, S., Grasso, M., Hannon, B., ... Van den Belt M. (1997). The value of the world's ecosystem services and natural capital. Nature, 387(6.630), 253-260. https://doi. org/10.1038/387253a0

Chen, N., Li, H., \& Wang, L. (2009). A

GIS-based approach for mapping direct use value of ecosystem services at a county scale: Management implications. Ecological economics, 68(11), 2.768-2.776. https://doi. org/10.1016/j.ecolecon.2008.12.001

Chung P., Sotomayor A., \& Lucero A. (2011). Diagnóstico del impacto de los productos forestales no madereros (PFNM) en el ingreso y alimentación de los pequeños propietarios en la región del Biobio, Chile. Instituto Forestal (INFOR), Ministerio de Agricultura de Chile.

Durán, A.P., Casalegno, S., Marquet, P.A., \& Gaston, K.J. (2013). Representation of ecosystem services by terrestrial protected areas: Chile as a case of study. PLoS One, 8(12), 1-8. https://doi.org/10.1371/ journal.pone. 0082643

Furci George-Nascimiento, G.M. (2007). Fungi Austral: Guía de campo de los hongos más vistosos de Chile. I.S.B.N: 978956-310-899-6. Recuperado de http:// fundacionphilippi.cl/sites/default/files/ fungi_austral.pdf

Gómez-Baggethun E., de Groot, R.S., Lomas, P.L., \& Montes, C. (2010). The history of ecosystem services in economic theory and practice: From early notions to markets and payment schemes. Ecological economics, 69(6), 1.209-1.218. https://doi. org/10.1016/j.ecolecon.2009.11.007 
Grêt-Regamey, A., Bebi, P., Bishop, I.D., \& Schimd, W.A. (2008). Linking GIS-based models to value ecosystem services in an Alpine region. Journal of environmental management, 89(3), 197-208. https://doi. org/10.1016/j.jenvman.2007.05.019

Haines-Young, R. \& Potschin, M. (2010). The links between biodiversity, ecosystem services and human well-being. In D.G. Raffaelli \& C.L.J. Frids (Eds.) Ecosystem ecology: a new synthesis. Cambridge, UK: Cambridge University Press.

Lara, A., Little, C., Urrutia, R., McPhee, J., Álvarez-Garretón, C., Oyarzún, C., ... Arismendi, I. (2009). Assessment of ecosystem services as an opportunity for the conservation and management of native forests in Chile. Forest ecology and management, 258(4), 415-424. https://doi. org/10.1016/j.foreco.2009.01.004

Luebert, F. \& Pliscoff, P. (2006). Sinopsis bioclimática y vegetacional de Chile. Editorial Universitaria. ISBN: 956-111832-7

Martín-López, B. \& Montes, C. (2010). Funciones y servicios de los ecosistemas: una herramienta para la gestión de los espacios naturales. Guía científica de Urdaibai, 1, 13-32. Recuperado de http:// www.ehu.eus/cdsea/web/wp-content/ uploads/2016/11/GuiaUrdaibaiC.pdf

Millennium Ecosystem Assessment (MA) (2005). Ecosystem and human well-being: Current states and trends. Washington DC: Island press. ISBN: 9781559632270

Nahuelhual, L., Carmona, A., Lozada, P., Jaramillo, A., \& Aguayo, M. (2013). Mapping recreation and ecotourism as a cultural ecosystem service: An application at the local level in Southern Chile. Applied geography, 40, 71-82. https://doi. org/10.1016/j.apgeog.2012.12.004

Nahuelhual, L., Carmona, A., Laterra, P., Barrena, J., \& Aguayo, M. (2014). A mapping approach to assess intangible cultural ecosystem services: The case of agriculture heritage in Southern Chile. Ecological indicators, 40, 90-101. https:// doi.org/10.1016/j.ecolind.2014.01.005

Núñez E., (2010). Método para la planificación delmanejo de áreas protegidas. Corporación Nacional Forestal (CONAF), Santiago, Chile.

Portela, R. \& Rademacher, I. (2001). A dynamic model of patterns of deforestation and their effect of the Brazilian Amazonia to provide ecosystem services. Ecological modelling, 143(1-2), 115-146. https://doi. org/10.1016/s0304-3800(01)00359-3

Valdebenito, G. (2012). Uso y valor de los productos forestales no madereros (PFNM) en Chile. (Informe INFOR). Recuperado de https://docplayer.es/83817994-Uso-y-valorde-los-productos-forestales-no-madererospfnm-en-chile.html

VanBeukering,P.J.H.,Cesar,H.J.S., \& Janssen, M.A. (2003). Economic valuation of the Leuser National Park on Sumatra, Indonesia. Ecological economics, 44(1), 43-62. https:// doi.org/10.1016/s0921-8009(02)00224-0

Villa, F., Bagstad, K.J, Voigt, B., Johnson, G.W., Portela, R., Honzák, M., \& Batker, D. (2014). A methodology for adaptable and robust ecosystem services assessment. PLoS One, 9(3), 1-18. https://doi.org/10.1371/ journal.pone.0091001 